\title{
Childhood Intestine Rotation Anomalies in Aba, Nigeria
}

\author{
Samuel Chidi Ekpemo ${ }^{1, *}$, Lisa Eweputanna ${ }^{2}$ Emeka Nkwo ${ }^{3}$ \\ ${ }^{1}$ Department of Surgery, Abia State University, Aba, Nigeria \\ ${ }^{2}$ Department of Radiology, Abia State University, Aba, Nigeria \\ ${ }^{3}$ Department of Obstetrics and Gynaecology, Federal Medical Center, Umuahia, Nigeria
}

Email address:

chidisamuelekpemo@gmail.com (S. C. Ekpemo)

*Corresponding author

\section{To cite this article:}

Samuel Chidi Ekpemo, Lisa Eweputanna, Emeka Nkwo. Childhood Intestine Rotation Anomalies in Aba, Nigeria. Advances in Surgical Sciences. Vol. 7, No. 1, 2019, pp. 1-4. doi: 10.11648/j.ass.20190701.11

Received: November 23, 2018; Accepted: January 21, 2019; Published: February 7, 2019

\begin{abstract}
Background: The anomalies of rotation, migration and fixation of the intestines are of intense interest to the pediatric surgeon, as they are frequently associated with midgut volvulus which has catastrophic consequences when diagnosis is delayed or even not considered. This study evaluates the outcomes of surgical management of intestinal malrotation in children. Materials and Methods: The medical records of all patients with symptomatic malrotation, who underwent surgery between January 2010 and September 2018, were reviewed. Patients' demographic characteristics, clinical features management, complications, and outcome were evaluated. Results: Ten patients (nine boys and a girl) underwent surgery for malrotation at a median age of 3 months. Eight presented with acute symptoms and two with chronic symptoms. All the patients had symptoms of intermittent or complete upper intestinal obstruction, and malrotation was documented by an upper gastrointestinal contrast study in six of them. Volvulus was found at the time of surgery in 5 patients, three of whom were neonates. Eight patients were treated by Ladd's operation. Median length of stay was 10 days. One patient with massive bowel gangrene due to volvulus had right hemicolectomy. There was two perioperative death from anastomostic leak and septicaemia with an overall mortality of $20 \%$. Conclusion: Bowel gangrene from volvulus contributes to mortality and small bowel adhesive intestinal obstruction is a cause of morbidity and mortality following surgery for intestinal malrotation. Neonates with bilious vomiting should raise the suspicion of malrotation until proven otherwise and given prompt intervention.
\end{abstract}

Keywords: Childhood, Intestine, Rotation, Anomalies

\section{Introduction}

Intestinal malrotation refers to all the abnormalities of intestinal rotation, migration and fixation of the midgut. The description of the embryological development of the intestine, the three arteries supplying a single tubular structure leading to the anatomical arrangement were all known the $19^{\text {th }}$ century [1-2]. The normal development of the human intestine involves two processes: rotation of the midgut and the subsequent fixation of the colon and mesentery. These processes occur in three stages. Stage 1 consists of umbilical cord herniation, lasting from approximately weeks 5 to 10 of embryonic development. The midgut lengthens disproportionately during this period and undergoes rotation around the superior mesenteric artery (SMA) axis for a total of $270^{\circ}$ in the counterclockwise direction. Stage 2 is the return of the midgut loop back into the abdomen; it occurs at approximately weeks 10 to 11. As the intestines re-enter the abdominal cavity, the cephalic midgut completes its $270^{\circ}$ counterclockwise rotation as the caudal midgut also completes its rotation, resulting in the duodenum coursing inferior and posterior to the SMA and the caecum located in the right lower quadrant. When completed, this rotation ensures that the attachment of the base of the midgut loop is spread along a diagonal stretching from the ligament of Trietz on the left upper quadrant to the ileocecal junction in the right lower quadrant of the abdomen. Stage 3 is the period of fixation, and lasts from the end of stage 2 until just after birth. The descending and ascending colon mesenteries fuse with the retroperitoneum, and the small bowel is fixed by a broad mesentery from the duodenojejunal 
junction in the left upper quadrant to the ileocecal valve in the right lower abdomen. The broad base of the small bowel mesentery stabilizes its position and prevents volvulus. The clinical presentation and surgical management were described by Dott, Ladd and Waugh with little progress made, apart from the recent new technology of diagnostic ultrasound and laparoscopy [35]. The incidence of symptomatic malrotation is reported to be 1 in 5000 live births [6].The incidence of this condition in African population is not known. However Amah [7] in Enugu South East Nigeria reported 8 cases over duration of 4 years while Ameh [8] in Zaria Northern Nigeria reported 14 cases over period of 18 years. More than half of the patients present with the symptoms during the first month of life, and virtually all have bile-stained vomiting. Because the consequences of malrotation associated with midgut volvulus may be so catastrophic, an understanding of the anatomy, diagnostic criteria, and appropriate therapy for this putative emergency illness is imperative [9]. This report reviews the pattern of clinical presentation and outcomes of surgical management of intestinal rotation anomalies at Abia State University Teaching Hospital Aba Nigeria.

\section{Methodology}

The records of ten pediatric patients with symptomatic malrotation of the intestine, seen from January 2010 to September 2018, were reviewed. Patient demographic characteristics, clinical presentation, imaging investigations, operations performed, complications, and outcomes were evaluated

\section{Results}

\subsection{Demography}

There were ten children (nine boys and one girl) of age in the range 5 days- 15 years (median: 20 days). Three patients (30\%) presented within the first 7 days of life, $5(50 \%)$ presented at less than 1 month of age, and $75 \%$ presented under 1 year of age. There were five termed neonates with weights ranging from 2.9 to $3.5 \mathrm{~kg}$ (mean: $4.2 \mathrm{~kg}$ ).

Table 1. Patient age at presentation.

\begin{tabular}{ll}
\hline Patient age at presentation & Number of patients \\
\hline 1 week & 3 \\
1 month & 5 \\
$<10$ years & 2 \\
\hline
\end{tabular}

\subsection{Clinical Features}

Preoperative symptoms included bilious emesis (80\%), fever $(50 \%)$, and abdominal pain (30\%). Bilious vomiting was the predominant sign in neonates. Abdominal pain followed by bilious emesis was evident in all the children older than 1 year of age.
Table 2. Clinical presentation.

\begin{tabular}{lll}
\hline Clinical features & Number of patients & Percentage \\
\hline Bilious emesis & 8 & $80 \%$ \\
Fever & 5 & $50 \%$ \\
Abdominal distension & 4 & $40 \%$ \\
Abdominal pain & 3 & $30 \%$ \\
Haematochezia & 2 & $20 \%$ \\
\hline
\end{tabular}

\subsection{Investigations}

Plain abdominal radiograph showed double bubble sign in five patients, dilated stomach, duodenum, and paucity of gas in the distal bowel suggesting duodenal obstruction. The Barium meal and follow through done showed malrotation in six patients and duodenal stenosis in two paients.

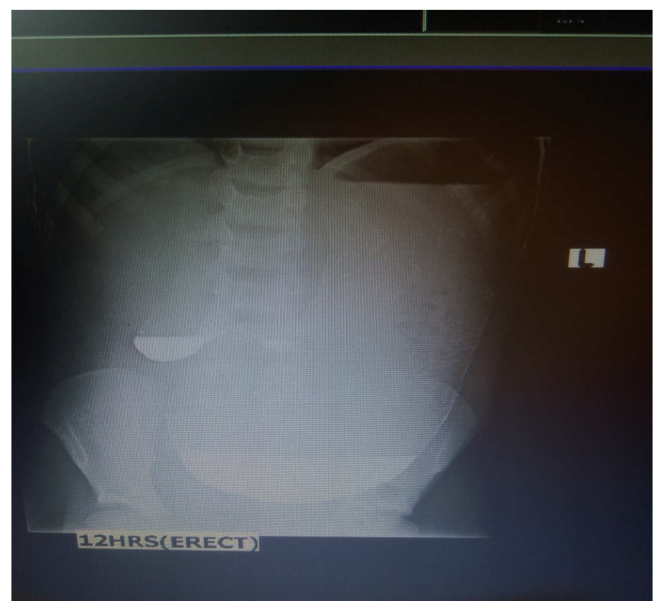

Figure 1. Barium meal and follow through.

AP view, There is marked gastric distension. The stomach extends to the pelvis. Poor gastric emptying is noted. Contrast is seen in the stomach at 12 hours. Contrast doesn't pass beyond the duodenum.

\subsection{Treatment}

Nine patients had emergency exploratory laparotomy and one was done electively. Intraoperative findings included Ladd's band in nine patients, five of whom were neonates. Nine patients were treated by Ladd's operation. One patient with massive gangrene of the small bowel due to volvulus had right hemicolectomy. A 6-year-old boy with duodenal obstruction from Ladd's band without volvulus had adhesiolysis. One patient developed anastomotic leak and one died of septicemia resulting in perioperative death. Two patients $(20 \%)$ were readmitted with small bowel intestinal obstruction within 2 months of initial discharge and they did well with non-operative management. Median hospital stay was 10 days (7-17 days).

\section{Discussion}

Malrotation is a spectrum of anatomic abnormalities of incomplete rotation and fixation of the intestinal tract during fetal development. Disorders of intestinal rotation and fixation are of paramount importance to the pediatric surgeon 
because they are most commonly seen in infancy and childhood and can have catastrophic consequences when midgut volvulus occurs. Early diagnosis and surgical treatment of this disorder can be life saving.

Majority of the patients $(50 \%)$ in this study presented during the neonatal period. This is in keeping with the literature reports of $50-80 \%$, but presentation can occur at any age. Males are said to be slightly more affected than females. The finding of nine boys against one girl is in keeping with this fact but is in contrast to the finding of equal sex distribution reported by Ameh in Zaria Northern Nigeria. [8]

The most dreaded complication of malrotation is volvulus [9]. Normal fixation of the midgut loop results in a broad diagonal attachment of the loop to the posterior abdominal wall, extending from the ligament of Trietz to the ileocecal junction. With malfixation, the distance between these two points of attachment may become shortened, leaving the midgut loop hanging on a narrow and unstable pedicle that easily predisposes to twisting (volvulus) and strangulation. Volvulus, a rotation of the gut along its mesenteric stalk occurs in $60-70 \%$ of neonates diagnosed with intestinal malrotation, with ultimate strangulation in about $15 \%$.[10] Delay in diagnosis may lead to intestinal ischemia, intestinal necrosis, septicemia, and short bowel syndrome.[11] A neonate in this report had volvulus, giving a volvulus rate of $10 \%$ in the neonates but it is lower than $14-42 \%$ in other reports.[8.10] This may be due to improved awareness of pediatric surgical services in this environment or difference in the number of patients reported in each series.

The clinical features in the neonates are indistinguishable from those of duodenal stenosis with bilious vomiting and epigastric distension which resolves after vomiting or aspiration by nasogastric tube [12]. Pain or irritability is not a prominent clinical feature in the neonate, but is a common feature in the toddler and older child [13]. Bilious vomiting in a neonate should raise the suspicion of intestinal malrotation with midgut volvulus until proven otherwise. Plain abdominal radiographs are often normal but may show a dilated duodenum with a fluid level and paucity of gas in the distal bowel as it was found in five patients in this series.

An upper gastrointestinal contrast study is the investigation of choice for any child presenting with bilious vomiting and should be done immediately. It is usually performed with barium, except in cases of a very sick infant or child in whom the presence of infarcted bowel or perforation is possible, in which case water-soluble contrast is used. It is important to document the first bolus of contrast medium through the duodenum in the anteroposterior as well as the lateral projection. This can be done by quickly rotating the patient to the lateral position once the duodenojejunal junction is reached. The main radiographic signs of malrotation are: $\bullet$ lateral radiograph suggesting that the distal duodenum is not attached in the retroperitoneum; low or medial position of the duodenojejunal junction; spiral "corkscrew" or Z-shaped course of the duodenum and proximal jejunum; and location of the proximal jejunum in the right abdomen. The contrast examination with a volvulus will show a dilated duodenum with a typical corkscrew appearance projecting forward away from the posterior abdominal wall on an oblique view. It was done in eight of our patients and it was suggestive of malrotation in six and duodenal stenosis in two cases. Assessment of the position and relationship between the superior mesenteric vessels by Doppler ultrasound is characteristic with the whirlpool appearance and may be a useful adjunct in the diagnosis of intestinal malrotation [14-16].

The pathologic effects of anomalies of rotation arise from excessive mobility, compression, or kinking of bowel and predisposition to torsion, volvulus, and intussusceptions from the narrow stalk [17-18]. Ladd's bands occurs when the caecum has failed to descend from the right upper quadrant to the right iliac fossa, anomalous fixation may occur, whereby dense fibrous bands (Ladd's bands) extend from the caecum and right colon across the duodenum to the retroperitoneum of the right upper quadrant. These bands may cause duodenal obstruction via extrinsic compression; however, the obstruction of the duodenum is most commonly caused by torsion at the base of the midgut mesentery. Bands may also form between the colon and the duodenum, drawing them closer together and predisposing the midgut towards volvulus. Ladd's procedure is the surgical treatment of choice for all malrotation. This involves derotation of the bowel if torsion is present, division of the peritoneal attachments lying across the duodenum from cecum to right upper quadrant, widening of the base of the mesentery, appendectomy, and taking down the ligament of Treitz and moving the duodenum to the right and, finally, returning the bowel to a position of nonrotation with the cecum placed into the left upper quadrant [5]. The morbidity and mortality of malrotation are mostly due to midgut vovulus and bowel adhesion. Delayed presentation may lead to bowel gangrene necessitating resection and anastomosis with a resultant increase in morbidity and mortality [10]. The perioperative mortality in this study was a result of anastomotic leak and septicemia.

Total parenteral nutrition is not readily available in most centers in low and middle income countries. Short gut syndrome secondary to bowel necrosis poses a great challenge [11]. If ischemic bowel is found at laparotomy from volvulus, every attempt should be made to preserve the bowel length and if any doubt exists about viability, a second-look laparotomy should be done 12-24 hours later without initial resection or with a very conservative resection. This has been shown to improve the outcomes. In this environment, the morbidity and mortality is compounded by delayed presentation and long referral chain [10]. The problems can be addressed by educating the parents, midwives, general practitioners, Obstetricians and pediatricians on the need for suspicion of malrotation in any child with bilious vomiting and prompt referral to centers where they can get appropriate treatment.

Two $(20 \%)$ patients were readmitted for adhesive intestinal obstruction in this report. This compares to $7-24 \%$ incidence 
of postoperative adhesions in previous reports [7-10]. The steps of the Ladd's procedure required much handling and manipulation of the bowel increasing the risk of adhesion. The adhesions resulting from the operation are thought to be advantageous because they are felt to stabilize the bowel in its new position. However, there have been controversies on fixation and non fixation of the bowel after Ladd's procedure with attendant merits and demerits [18-21]. The newly evolving laparoscopic approach for Ladd's procedure may reduce the incidence of postoperative adhesions [5]. However, laparoscope is not readily available in low and middle income countries like Nigeria. The mortality from malrotation has been reported to be $6.9-16.7 \%$ compared to perioperative mortality of $20 \%$ in this report.

\section{Conclusion}

The report a $20 \%$ risk of postoperative bowel obstruction after a Ladd's procedure and $20 \%$ overall mortality in this series. A high index of suspicion in the neonates with bilious vomiting needs rapid diagnosis and appropriate operative therapy results in a favorable outcome. Malrotation with its propensity for volvulus is no doubt a catastrophe lying within the abdomen. Detailed education about the potential for postoperative small bowel obstruction and the need for early presentation must be given to the parents of these children on discharge

\section{References}

[1] Mall FP. Development of human intestine and its position in adult. John Hopkins Hosp B 1896; 9:197-208.

[2] Filston HC, Kirk DR. Malrotation, the ubiquitous anomaly. J pediatr Surg 1981; 16:614-620.

[3] Dott NM. Anomalies of intestinal rotation: their embryology and surgical aspects with report of 5 cases. Br J Surg 1923; 11: $253-286$

[4] Ladd WE. Congenital obstruction of the duodenum in children. N Engl J Medicine 1932;206:297-283.

[5] Gross E, Chen MK, Lobe TE: Laparoscopic evaluation and treatment of intestinal malrotation in infants. Surg Endosc 1996;10:936-937.

[6] Strouse PJ. Disorders of intestinal rotation and fixation ("malrotation"). Pediatr Radiol 2004; 34:837-851
[7] Amah CC, Agugua-Obianyo NEN, Ekenze SO. Intestinal malrotation in the older child: common diagnostic pitfalls. W Afr J Radiol 2004; 11:33-37

[8] Ameh EA, Chirdan LB. Intestinal Malrotation: Experience in Zaria, Nigeria. WAJM 2001; 20, 227-30

[9] Torres AM, Ziegler MM. Malrotation of the Intestine. World J Surg. 1993;17: 326-

[10] Ameh EA, Nmadu PT. Intestinal volvulus:aetiology,morbidity and mortality in Nigerian children.Pediatr Surg Int 2000;16:50-52.

[11] Reyes J, Bueno J, Kocoshis S, et al: Current status of intestinal transplantation in children. $\mathrm{J}$ Pediatr Surg 1998;33:243-254.

[12] Bonadio WA, Clarkson T, Naus J. The clinical features of children with malrotation of the intestine. Pediatr Emerg Care $1991 ; 7: 349$

[13] Rescorla FJ, Shedd FJ, Grosfeld JL, Vane DW, West KW: Anomalies of intestinal rotation in childhood: anomalies of 447 cases. Surgery 1990; 108:710-716.

[14] Prarcos D, Sann L, Gein G, et al: Ultrasound diagnosis of midgut volvulus: the whirlpool sign. Pediatr Radiol 1992;22:18-20.

[15] Dufour D, Delaet MH, Dassonville M, Cadranel S, Perlmutter $\mathrm{N}$ : Midgut malrotation, the reliability of sonographic diagnosis. Pediatr Radiol 1992; 22:21-23

[16] Ashley LM, Allen S, Teele RL: A normal sonogram does not exclude malrotation. Pediatr Radiol 2001; 31:354-356.

[17] Waugh GEA: The morbid consequences of a mobile ascending colon. Br J Surg 1920; 7:343,

[18] Brerefon RJ, Taylor B, Hall CM: Intussception and intestinal malrotation in infants: Waugh's syndrome. Br J Surg 1986; 73:55-57.

[19] Spitz L, Orr JD, Harries JT: Obstructive jaundice secondary to chronic midgut volvulus. Arch Dis Child 1983;58:383-385.

[20] Schwartz DL, So HB, Schneider KM, Becker JM: Recurrent chylous ascites associated with intestinal malrotation and lymphatic rupture. J Pediatr Surg 1983; 18:177-179.

[21] Stauffer UG, Hermann P: Comparison of late results in patients with corrected intestinal malrotation with and without fixation of the mesentery. J Pediatr Surg 1980; 15:1.

[22] Brennon WS, Bill AH: Prophylactic fixation of the intestine for midgut non-rotation. Surg Gynecol Obstet 1974;138:181184. 